\title{
Health Education
}

National Cancer Institute

\section{Source}

National Cancer Institute. Health Education. NCI Thesaurus. Code C16664.

Health information provided to individuals or communities designed to reduce ill health and enhance good health by influencing beliefs, attitudes and behavior. 